\title{
DEEP PLACEMENT OF NPK BRIQUETTE IN RICE: ENVIRONMENT FRIENDLY TECHNOLOGY FOR SUSTAINABLE RICE PRODUCTION
}

\author{
A.B.S. Sarker ${ }^{1}$, A.J. Mridha ${ }^{2}$ and M.A. Mazid Miah ${ }^{3}$ \\ ${ }^{1}$ PSO, Agronomy Division; ${ }^{2}$ Agronomy Division, ${ }^{3}$ Ex Head, Soil Science Division \\ Bangladesh Rice Research Institute, Gazipur \\ Corresponding E-mail: sarkerabs@yahoo.com
}

(Received: 05 December 2021, Accepted: 15 December 2021)

Keyword: USG, Boro season, T. Aman season, T. Aus season

\begin{abstract}
Nine experiments were conducted at the Bangladesh Rice Research Institute (BRRI) farm, Gazipur, BRRI regional station, Sagardi, Barisal and farmers' field of Barisal during Boro, T. Aus and T. Aman season, 2012 to evaluate the NPK briquette efficacy in rice production. Experimental results revealed that deep placement of NPK briquette $(2 \mathrm{x}$ $2.4 \mathrm{~g}$ ) increased rice grain yield about $10 \%$ and it saves 51, 9 and $20 \mathrm{~kg}^{\mathrm{NPK}} \mathrm{ha}^{-1}$ than BRRI fertilizer recommended rate in Boro season. Similarly, NPK briquette $(1 \times 3.4 \mathrm{~g})$ produced 28 and $18 \%$ more rice grain yield than BRRI fertilizer recommended rate with a savings of $29 \mathrm{~kg} \mathrm{~N}$ and $7 \mathrm{~kg} \mathrm{~K} \mathrm{ha}^{-1}$ in T. Aus and T. Aman season, respectively. In general, deep placement method gave higher grain yield compared to conventional urea application. Undiscounted benefit cost ratio (BCR) was 1.44, 2.40 and 3.20 for Boro, T. Aus and T. Aman, respectively when comparing at the same rates of $\mathrm{N}$ application. Thus, use of NPK briquette over urea broadcast and incorporation was economically viable and efficient for rice cultivation. This method helps to reduce $\mathrm{N}$ loss through soil-water interface which plays a vital role in reducing environmental pollution.
\end{abstract}

\section{Introduction}

Rice is the most extensively cultivated crop in Bangladesh and staple food of Bangladeshi people also. Bangladesh is an agro-based country and predominantly rice based one which earns about $21.10 \%$ of her gross domestic product (GDP) from agriculture (Anonymous, 2008). The area and the production of rice in this country are 11.25 million hectares and 29.75 million tons, respectively (AIS, 2008).

The edaphic and climatic factors of Bangladesh are favorable for rice cultivation but the average yield of Boro rice in this country is quite low $\left(2.55 \mathrm{t} \mathrm{ha}^{-1}\right)$ in the fiscal year of 2006-2007 (BBS, 2007). The farmers have to produce maximum rice from minimum land by adopting improved agronomic practices. Deep placement of NPK briquette can play a significant role in increasing yield of Boro rice. Bhuiyan et al. (1988) reported that deep point placement of USG resulted in significantly higher grain yield of rice than split application of prilled urea. Further increases in grain yield, better $\mathrm{N}$ use efficiency $(\mathrm{kg}$ grain $/ \mathrm{kg} \mathrm{N})$ and higher apparent $\mathrm{N}$ recovery occurred when the hole was closed after USG application. Similarly, Mohanty et al. (1999) reported that USG deep placement resulted additional grain yield of 1080,510 and $350 \mathrm{~kg} / \mathrm{ha}$ over prilled urea in alternate wetting and drying, shallow low land and intermediate low land, respectively.

Recent increase in the cost of inorganic fertilizer makes it important to examine the relationships between grain yield and $\mathrm{N}$ utilization by rice to improve the efficiency of $\mathrm{N}$ utilization. Many strategies have been developed to increase the efficiency of urea-N use from fertilizer through proper timing, rate, deep placement, modified forms of fertilizer, and use of nitrification and urease inhibitors. Among them deep placement of urea fertilizer is one of the most effective application method in reducing total 
$\mathrm{N}$ loss in the floodwater and is likely to minimize losses through volatilization and surface runoff (De Datta \& Craswell, 1982). Similarly, Kapoor et al. (2008) reported that broadcast application of N as urea resulted in an average ten times higher amounts of ammonium $\mathrm{N}$ in flood water compared to deep placement of urea briquette. They also reported that deep placement of $\mathrm{P}$ and $\mathrm{K}$ resulted in lower content of $\mathrm{P}$ and $\mathrm{K}$ in floodwater.

In rice cultivation system generally, $\mathrm{P}$ and $\mathrm{K}$ fertilizer is applied as broadcast but an NPK briquette is applied by deep point placement. For very low level of $\mathrm{P}$ and $\mathrm{K}$ in soil, particularly under upland condition, initial broadcast application followed by deep point placement to each subsequent planting is generally recommended. The use of urea briquettes gave higher rice yields and resulted in greater removal of NPK than prilled urea (Prasad and Prasad, 1983). Kapoor et al. (2008) reported that deep placed N-P briquettes gave significantly higher rice grain yield, total $\mathrm{P}$ and $\mathrm{K}$ uptake. They also found that closer spacing $(20 \mathrm{~cm} \times 10 \mathrm{~cm})$ led to better utilization of $\mathrm{P}$ and $\mathrm{K}$ and provided opportunities for deep placement of N-P or N-P-K briquettes in soils with low available P. Bulbule et al. (2008) reported that grain yield of rice significantly increased when the crop was fertilized through briquettes (56-30-30 $\mathrm{kg} \mathrm{NPK} / \mathrm{ha}$ ) as compared to the application of conventional fertilizers (100-50-50 kg NPK/ha). The deep placement of NPK briquette combines the concept of balance fertilization and deep placement advantage towards higher nutrient use efficiency in rice. In this context, NPK briquette deep placement technology for higher rice yield as well as better utilization of applied fertilizer by the crop and indirectly to avoid environmental pollution through volatilization and surface runoff to be ascertained.

\section{Materials and Methods}

Nine field experiments were conducted at BRRI farm Gazipur, BRRI Regional Station, Sagordi, Barisal and farmer's field at Babuganj of Barisal by Agronomy division of BRRI. Eight treatments were tested. The treatments were $\mathrm{T}_{1}=\mathrm{BRRI}$ recommended dose of prilled urea at three splits and P K $\mathrm{S}$ and $\mathrm{Zn}$ fertilizer was applied as basal at final land preparation, $\mathrm{T}_{2}=$ One $2.70 \mathrm{~g} \mathrm{USG}\left(\mathrm{N}_{78}\right)$ in Boro and one $1.90 \mathrm{~g} \mathrm{USG}\left(\mathrm{N}_{52}\right)$ was placed at the centre of each four hills + BRRI recommended rate P K S and $\mathrm{Zn}$ fertilizer was applied as basal at final land preparation, $\mathrm{T}_{3}=2.40 \mathrm{~g}$ NPK briquette (two in Boro, one in T. Aus and T. Aman) was placed at the centre of each four hills (N:P:K ratio = 7.0:1.6:2.0 and doses of NPK $=87 \mathrm{~kg} \mathrm{Nha}^{-1}, 20 \mathrm{~kg} \mathrm{Pha}^{-1}$ and $25 \mathrm{~kg} \mathrm{Kha}^{-1}$ in Boro and $43.5 \mathrm{~N} \mathrm{~kg} \mathrm{Nha}^{-1}, 10 \mathrm{~kg} \mathrm{Pha}{ }^{-1}$ and $12.5 \mathrm{~kg} \mathrm{Kha}^{-1}$ in T Aus and T. Aman, $\mathrm{S}$ and $\mathrm{Zn}$ as recommended, $\mathrm{T}_{4}=$ One $3.40 \mathrm{~g}$ NPK briquette was placed at the centre of each four hills (N:P:K ratio $=9.1: 2.4: 3.5$ and doses of NPK $=57 \mathrm{~kg} \mathrm{Nha}^{-1}$, $15 \mathrm{~kg} \mathrm{Pha}^{-1}$ and $22 \mathrm{~kg} \mathrm{Kha}^{-1}$ ) and $\mathrm{S}$ and $\mathrm{Zn}$ as recommended, $\mathrm{T}_{5}=$ NPKS and $\mathrm{Zn}$ doses were same as $\mathrm{T}_{3}$ but sources were Urea, TSP, MP, $\mathrm{T}_{6}=$ NPKS and $\mathrm{Zn}$ doses were same as $\mathrm{T}_{4}$ but sources were Urea, TSP, MoP, $\mathrm{T}_{7}=$ Absolute Control and $\mathrm{T}_{8}=\mathrm{N}$ alone for prilled urea. The nutrients of the treatments for the location and season are given in Table 1. The experiment was laid down in RCB design with three replications. The tested variety was BRRI dhan29 in Boro, BRRI dhan27 in T Aus and BRRI dhan49 in T. Aman season. The unit plot size was 4 × $6 \mathrm{~m}$. Transplanting was done by two seedlings per hill and the seedling age was 40, 30 and 35 days, respectively in Boro, T. Aus and T. Aman season at plant spacing of $20 \mathrm{~cm} \times 20 \mathrm{~cm}$. All fertilizer was applied as per treatment before and after transplanting. The USG and NPK briquettes were deep placed between four hills as per treatment after 7 days of transplanting.

Table 1. Summary of treatments applied at different locations and across seasons

\begin{tabular}{lcccccc}
\hline Treatments & Description & Location & Season & $\begin{array}{c}\text { N Rate } \\
(\mathbf{k g} / \mathbf{h a})\end{array}$ & $\begin{array}{c}\text { P Rate } \\
(\mathbf{k g} / \mathbf{h a})\end{array}$ & $\begin{array}{c}\text { K Rate } \\
(\mathbf{k g} / \mathbf{h a})\end{array}$ \\
\hline $\mathrm{T}_{1}=$ Rec. fertilizer rate & BRRI & On-Station & Boro & $138^{\mathrm{a}}$ & $26^{\mathrm{b}}$ & $75^{\mathrm{b}}$ \\
& Recommend & (Gazipur) $)$ & Aus & $77^{\mathrm{a}}$ & $15^{\mathrm{b}}$ & $37^{\mathrm{b}}$ \\
& & & Aman & $93^{\mathrm{a}}$ & $16^{\mathrm{b}}$ & $42^{\mathrm{b}}$ \\
& & On-Station & Boro & 138 & 30 & 30 \\
& & (Barisal) & Aus & 77 & 11 & 17 \\
& & & Aman & 93 & 15 & 17 \\
& & On-Farm & Boro & 138 & 30 & 30
\end{tabular}




\begin{tabular}{|c|c|c|c|c|c|c|}
\hline & & (Babuganj) & $\begin{array}{c}\text { Aus } \\
\text { Aman } \\
\end{array}$ & $\begin{array}{l}77 \\
93 \\
\end{array}$ & $\begin{array}{l}11 \\
15 \\
\end{array}$ & $\begin{array}{l}18 \\
18 \\
\end{array}$ \\
\hline $\begin{array}{l}\mathrm{T}_{2}=\mathrm{USG} \mathrm{N}_{78} \mathrm{~kg} \mathrm{ha}^{-1}+ \\
\text { Rec. PKSZn }\end{array}$ & $\begin{array}{l}\text { USG (one- } 2.7 \mathrm{~g} \\
\text { briq) }\end{array}$ & All & All & $78^{\mathrm{c}}$ & \multirow{2}{*}{\multicolumn{2}{|c|}{$\begin{array}{l}\text { PK rate similar as } T_{1} \\
\text { in respective season }\end{array}$}} \\
\hline $\begin{array}{l}\mathrm{T}_{2}=\mathrm{USG} \mathrm{N}_{52} \mathrm{~kg} \mathrm{ha}^{-1}+ \\
\text { Rec. PKSZn }\end{array}$ & $\begin{array}{l}\text { USG (one-1.8 g } \\
\text { briq) }\end{array}$ & All & $\begin{array}{l}\text { T. Aus and } \\
\text { T. Aman }\end{array}$ & 52 & & \\
\hline $\begin{array}{l}\mathrm{T}_{3}=(2 \times 2.40) \mathrm{NPK} \\
\text { briquette } \mathrm{N}_{87}\end{array}$ & $\begin{array}{l}\text { NPK (two-2.4 g } \\
\text { briq) }\end{array}$ & All & Boro & $87^{c}$ & $20^{c}$ & $25^{\mathrm{c}}$ \\
\hline $\begin{array}{l}\mathrm{T}_{3}=(1 \times 2.40) \mathrm{NPK} \\
\text { briequette } \mathrm{N}_{44}\end{array}$ & $\begin{array}{c}\text { NPK (one-2.4 g } \\
\text { briq) }\end{array}$ & All & $\begin{array}{l}\text { T. Aus and } \\
\text { T. Aman }\end{array}$ & $43.5^{c}$ & $10^{\mathrm{c}}$ & $12.5^{\mathrm{c}}$ \\
\hline $\begin{array}{l}\mathrm{T}_{4}=3.40 \mathrm{~g} \mathrm{NPK} \\
\text { briequette } \mathrm{N}_{57}\end{array}$ & $\begin{array}{l}\text { NPK (one-3.4 g } \\
\text { briq) }\end{array}$ & All & All & $57^{c}$ & $15^{\mathrm{c}}$ & $22^{c}$ \\
\hline \multirow[t]{2}{*}{$\begin{array}{l}\mathrm{T}_{5}=\mathrm{NPK} \text { same at } \mathrm{T}_{3} \text { from } \\
\text { urea, TSP and MoP }\end{array}$} & $\begin{array}{c}\text { NPK (urea,TSP, } \\
\text { MoP) }\end{array}$ & All & Boro & $87^{\mathrm{a}}$ & $20^{\mathrm{b}}$ & $25^{\mathrm{b}}$ \\
\hline & $\begin{array}{l}\text { NPK (urea, TSP, } \\
\text { MoP) }\end{array}$ & All & $\begin{array}{l}\text { T. Aus and } \\
\text { T. Aman }\end{array}$ & $43.5^{\mathrm{a}}$ & $10^{\mathrm{b}}$ & $12.5^{\mathrm{b}}$ \\
\hline $\begin{array}{l}\mathrm{T}_{6}=\mathrm{NPK} \text { same at } \mathrm{T}_{4} \text { from } \\
\text { urea, TSP and MoP }\end{array}$ & $\begin{array}{c}\text { NPK (urea, TSP, } \\
\text { MoP) }\end{array}$ & All & All & $57^{\mathrm{a}}$ & $15^{\mathrm{b}}$ & $22^{b}$ \\
\hline $\mathrm{T}_{7}=$ Abs. control & Absolute Control & All & All & 0 & 0 & 0 \\
\hline $\mathrm{T}_{8}=$ Only $\mathrm{N}$ & $\mathrm{N}$ alone & All & All & 102 & $25^{\mathrm{d}}$ & $64^{\mathrm{d}}$ \\
\hline
\end{tabular}

Placement of USG and NPK briquette was done 10 days after transplanting. Flood water $\mathrm{NH}_{4}-\mathrm{N}$ was determined through Spectrophotometer by using Nessler Reagent (Radov et al., 1985) and continued for seven days. Grain yield ( $\mathrm{t} / \mathrm{ha}$ ) was determined from $5 \mathrm{~m}^{2}$ from each treatment and straw yield per square meter was calculated from 16 hills. The data were statistically analyzed.

Table 2. Treatment description for Boro rice

\begin{tabular}{clcccccc}
\hline Treatments & \multicolumn{1}{c}{ Description } & $\begin{array}{c}\text { N Rate } \\
(\mathrm{kg} / \mathrm{ha})\end{array}$ & $\begin{array}{c}\text { P Rate } \\
(\mathrm{kg} / \mathrm{ha})\end{array}$ & $\begin{array}{c}\mathrm{K} \text { Rate } \\
(\mathrm{kg} / \mathrm{ha})\end{array}$ & $\begin{array}{c}\text { Basal /deep } \\
\text { placed N } \\
(\mathrm{kg} / \mathrm{ha})\end{array}$ & $\begin{array}{c}1^{\text {st }} \text { Top- } \\
\text { Dress N } \\
(\mathrm{kg} / \mathrm{ha})\end{array}$ & $\begin{array}{c}2^{\text {nd }} \text { Top- } \\
\text { Dress N } \\
(\mathrm{kg} / \mathrm{ha})\end{array}$ \\
\hline $\mathrm{T}_{1}$ & Check & 0 & $25^{\mathrm{a}}$ & $64^{\mathrm{b}}$ & 0 & 0 & 0 \\
$\mathrm{~T}_{2}$ & USG (one-2.7 g briq) & 78 & $25^{\mathrm{a}}$ & $64^{\mathrm{b}}$ & 78 & 0 & 0 \\
$\mathrm{~T}_{3}$ & USG (two-1.8 g briq) & 104 & $25^{\mathrm{a}}$ & $64^{\mathrm{b}}$ & 104 & 0 & 0 \\
$\mathrm{~T}_{4}$ & Urea & 78 & $25^{\mathrm{a}}$ & $64^{\mathrm{b}}$ & 26 & 26 & 26 \\
$\mathrm{~T}_{5}$ & Urea & 104 & $25^{\mathrm{a}}$ & $64^{\mathrm{b}}$ & 34 & 35 & 35 \\
$\mathrm{~T}_{6}$ & NPK (two-2.4 g briq) & 78 & $16^{\mathrm{c}}$ & $42^{\mathrm{c}}$ & 78 & 0 & 0 \\
$\mathrm{~T}_{7}$ & Urea & 156 & $25^{\mathrm{a}}$ & $64^{\mathrm{b}}$ & 52 & 52 & 52 \\
$\mathrm{~T}_{8}$ & NPK (two-3.4 g briq) & 102 & $25^{\mathrm{d}}$ & $64^{\mathrm{d}}$ & 102 & 0 & 0 \\
\hline
\end{tabular}

${ }^{\mathrm{a}}$ Applied as TSP, ${ }^{\mathrm{b}}$ Applied as $\mathrm{MoP}(\mathrm{KCl}),{ }^{\mathrm{c}} \mathrm{P}$ and $\mathrm{K}$ is applied as NPK briquette

(Treat. 6; made with $45 \mathrm{~kg}$ Urea $+27 \mathrm{~kg}$ DAP $+28 \mathrm{~kg} \mathrm{MoP}),{ }^{\mathrm{d}} \mathrm{P}$ and $\mathrm{K}$ is applied as NPK briquette (Treatment 8; made with $40 \mathrm{~kg}$ Urea $+30 \mathrm{~kg}$ DAP $+30 \mathrm{~kg}$ MoP)

Table 3. Treatment description for T. Aus and T. Aman rice

\begin{tabular}{clcccccc}
\hline Treatments & \multicolumn{1}{c}{ Description } & $\begin{array}{c}\text { N Rate } \\
\text { (kg/ha) }\end{array}$ & $\begin{array}{c}\text { P Rate } \\
\text { (kg/ha) }\end{array}$ & $\begin{array}{c}\text { K Rate } \\
(\mathbf{k g} / \mathbf{h a})\end{array}$ & $\begin{array}{c}\text { Basal /deep } \\
\text { placed N } \\
\text { (kg/ha) }\end{array}$ & $\begin{array}{c}\mathbf{1}^{\text {st }} \text { Top- } \\
\text { Dress N } \\
\text { (kg/ha) }\end{array}$ & $\begin{array}{c}\mathbf{2}^{\text {nd }} \text { Top- } \\
\text { Dress N } \\
\text { (kg/ha) }\end{array}$ \\
\hline $\mathrm{T}_{1}$ & Check & 0 & $16^{\mathrm{a}}$ & $42^{\mathrm{b}}$ & 0 & 0 & 0 \\
$\mathrm{~T}_{2}$ & USG (one-1.8 g briq) & 52 & $16^{\mathrm{a}}$ & $42^{\mathrm{b}}$ & 52 & 0 & 0 \\
$\mathrm{~T}_{3}$ & USG (one-2.7 g briq) & 78 & $16^{\mathrm{a}}$ & $42^{\mathrm{b}}$ & 78 & 0 & 0 \\
$\mathrm{~T}_{4}$ & Urea & 78 & $16^{\mathrm{a}}$ & $42^{\mathrm{b}}$ & 26 & 26 & 26 \\
$\mathrm{~T}_{5}$ & UDP (two-1.8 g briq) & 104 & $16^{\mathrm{a}}$ & $42^{\mathrm{b}}$ & 104 & 0 & 0 \\
$\mathrm{~T}_{6}$ & NPK (one-3.4 g briq) & 51 & $13^{\mathrm{c}}$ & $32^{\mathrm{c}}$ & 51 & 0 & 0
\end{tabular}




\begin{tabular}{|c|c|c|c|c|c|c|c|}
\hline $\mathrm{T}_{7}$ & Urea & 120 & $16^{\mathrm{a}}$ & $42^{\mathrm{b}}$ & 40 & 40 & 40 \\
\hline $\mathrm{T}_{8}$ & NPK (two- $2.4 \mathrm{~g}$ briq) & 78 & $16^{\mathrm{d}}$ & $42^{\mathrm{d}}$ & 78 & 0 & 0 \\
\hline
\end{tabular}

${ }^{\mathrm{a}}$ Applied as TSP, ${ }^{\mathrm{b}}$ Applied as $\mathrm{MoP}(\mathrm{KCl}),{ }^{\mathrm{c}} \mathrm{P}$ and $\mathrm{K}$ is applied as NPK briquette (Treat. 6; made with $45 \mathrm{~kg}$ Urea $+27 \mathrm{~kg}$ DAP $+28 \mathrm{~kg} \mathrm{MoP}),{ }^{\mathrm{d}} \mathrm{P}$ and $\mathrm{K}$ is applied as NPK briquette (Treatment 8 ; made with $40 \mathrm{~kg}$ Urea $+30 \mathrm{~kg}$ DAP $+30 \mathrm{~kg} \mathrm{MoP})$

\section{Results and Discussion}

Grain yield of nine locations with all treatments of Boro, T. Aus and T. Aman are presented in Table 46 and grain yield advantage (\%) over recommended fertilizer rate is shown in Table 7. BRRI recommended fertilizer rate (NPKSZn) produced higher grain yield than without fertilizer and $\mathrm{N}$ alone treatment irrespective of locations and seasons. The only exception was observed for the first cropping (Boro) at Babuganj where $\mathrm{N}$ alone treatment with available soil $\mathrm{P}$ and $\mathrm{K}$ gave similar yield as the BRRI recommended treatment. Overall, the highest grain yields were obtained with the deep placement of NPK briquette at all locations. During the Boro season $\mathrm{T}_{3}$ (two-2.4 g NPK briquette) which was three locations average $10.06 \%$ higher. During the T. Aus and T. Aman season $\mathrm{T}_{4}$ (one-3.4 g NPK briquette) gave the highest grain yield and it was three locations average $17.69 \%$ higher in T Aus and $27.99 \%$ in T Aman season (Table 7).

Table 4. Effect of different treatments on the grain yield of BRRI dhan29 during Boro season 2012 at different locations

\begin{tabular}{|c|c|c|c|}
\hline \multirow[t]{2}{*}{ Treatments } & \multicolumn{3}{|c|}{ Boro } \\
\hline & $\begin{array}{l}\text { On-Station } \\
\text { (Gazipur) }\end{array}$ & $\begin{array}{c}\text { On-Station } \\
\text { (Barisal) }\end{array}$ & On-Farm (Barisal) \\
\hline$T_{1}=$ Rec. fertilizer rate & $7.00 \mathrm{~b}$ & $6.99 \mathrm{ab}$ & $6.67 \mathrm{bc}$ \\
\hline $\mathrm{T}_{2}=\mathrm{USG} \mathrm{N}_{52} \mathrm{~kg} \mathrm{ha}^{-1}+$ Rec. PKSZn & $7.13 \mathrm{ab}$ & $7.15 \mathrm{a}$ & $7.03 \mathrm{~b}$ \\
\hline $\mathrm{T}_{3}=(2 \times 2.40) \mathrm{NPK}$ briqutte $\mathrm{N}_{87}$ & $7.47 \mathrm{a}$ & $7.29 \mathrm{a}$ & $7.94 \mathrm{a}$ \\
\hline $\mathrm{T}_{4}=3.40 \mathrm{~g} \mathrm{NPK}$ briqutte $\mathrm{N}_{57}$ & $5.92 \mathrm{c}$ & $6.43 \mathrm{~b}$ & $6.13 \mathrm{c}$ \\
\hline $\begin{array}{l}\mathrm{T}_{5}=\mathrm{NPK} \text { same at } \mathrm{T}_{3} \text { from urea, TSP } \\
\text { and MoP }\end{array}$ & $6.96 \mathrm{~b}$ & $6.89 \mathrm{ab}$ & $7.16 \mathrm{~b}$ \\
\hline $\begin{array}{l}\mathrm{T}_{6}=\mathrm{NPK} \text { same at } \mathrm{T}_{4} \text { from urea, TSP } \\
\text { and MoP }\end{array}$ & $6.12 \mathrm{c}$ & $6.26 \mathrm{~b}$ & $6.22 \mathrm{c}$ \\
\hline $\mathrm{T}_{7}=$ Absolute control & $3.89 \mathrm{~d}$ & $3.20 \mathrm{c}$ & $4.14 \mathrm{~d}$ \\
\hline $\mathrm{T}_{8}=$ Only N & $5.88 \mathrm{c}$ & $6.19 \mathrm{~b}$ & $6.88 \mathrm{bc}$ \\
\hline
\end{tabular}

Figures in a column means followed by different letters differ significantly, whereas figure with common letter(s) are not significantly different at the 5\% level by DMRT

Table 5. Effect of different treatments on the grain yield of BRRI dhan49 during T Aman season 2012 at different locations

\begin{tabular}{lccc}
\hline Treatments & \multicolumn{2}{c}{ T. Aman } \\
\cline { 2 - 4 } & $\begin{array}{c}\text { On-Station } \\
\text { (Gazipur) }\end{array}$ & $\begin{array}{c}\text { On-Station } \\
\text { (Barisal) }\end{array}$ & $\begin{array}{c}\text { On-Farm } \\
\text { (Barisal) }\end{array}$ \\
\hline $\mathrm{T}_{1}=$ Rec. fertilizer rate & $4.20 \mathrm{bc}$ & $4.77 \mathrm{bc}$ & $4.40 \mathrm{~b}$ \\
$\mathrm{~T}_{2}=$ USG N52 $\mathrm{kg} \mathrm{ha}^{-1}+$ Rec. PKSZn & $4.44 \mathrm{~b}$ & $5.26 \mathrm{ab}$ & $4.94 \mathrm{ab}$ \\
$\mathrm{T}_{3}=(1 \times 2.40)$ NPK briqutte N44 & $3.89 \mathrm{c}$ & $5.54 \mathrm{ab}$ & $4.22 \mathrm{~b}$ \\
$\mathrm{~T}_{4}=$ 3.40 g NPK briqutte $\mathrm{N}_{57}$ & $4.85 \mathrm{a}$ & $5.70 \mathrm{a}$ & $5.19 \mathrm{a}$ \\
$\mathrm{T}_{5}=$ NPK same at T $\mathrm{T}_{3}$ from urea, TSP and MoP & $3.27 \mathrm{~d}$ & $4.68 \mathrm{bc}$ & $4.01 \mathrm{bc}$ \\
$\mathrm{T}_{6}=$ NPK same at $\mathrm{T}_{4}$ from urea, TSP and MoP & $4.03 \mathrm{~d}$ & $4.70 \mathrm{bc}$ & $4.37 \mathrm{~b}$ \\
$\mathrm{~T}_{7}=$ Absolute control & $2.96 \mathrm{e}$ & $3.60 \mathrm{~d}$ & $3.16 \mathrm{~d}$ \\
$\mathrm{~T}_{8}=$ Only N & $3.30 \mathrm{~d}$ & $4,29 \mathrm{~cd}$ & $3.55 \mathrm{~cd}$ \\
\hline
\end{tabular}


Figures in a column means followed by different letters differ significantly, whereas figure with common letter(s) are not significantly different at the $5 \%$ level by DMRT

Table 6. Effect of different treatments on the grain yield of BRRI dhan27 during T.Aus season 2012 at different locations

\begin{tabular}{|c|c|c|c|}
\hline \multirow[t]{2}{*}{ Treatments } & \multicolumn{3}{|c|}{ T. Aus } \\
\hline & $\begin{array}{c}\text { On -Station } \\
\text { (Gazipur) }\end{array}$ & $\begin{array}{c}\text { On- Station } \\
\text { (Barisal) }\end{array}$ & $\begin{array}{l}\text { On-Farm } \\
\text { (Barisal) }\end{array}$ \\
\hline $\mathrm{T}_{1}=$ Rec. fertilizer rate & $3.24 \mathrm{c}$ & $2.84 \mathrm{~d}$ & $2.73 \mathrm{~b}$ \\
\hline $\mathrm{T}_{2}=$ USG N$_{52} \mathrm{~kg} \mathrm{ha}^{-1}+$ Rec. PKSZn & $3.57 \mathrm{~b}$ & $3.34 \mathrm{~b}$ & $2.90 \mathrm{~b}$ \\
\hline $\mathrm{T}_{3}=(1 \times 2.40)$ NPK briquette $\mathrm{N}_{44}$ & $3.20 \mathrm{c}$ & $3.10 \mathrm{c}$ & $2.96 \mathrm{~b}$ \\
\hline $\mathrm{T}_{4}=3.40 \mathrm{~g}$ NPK briquette $\mathrm{N}_{57}$ & $3.93 \mathrm{a}$ & $3.58 \mathrm{a}$ & $3.72 \mathrm{a}$ \\
\hline $\mathrm{T}_{5}=\mathrm{NPK}$ same at $\mathrm{T}_{3}$ from urea, $\mathrm{TSP}$ and $\mathrm{MoP}$ & $2.96 \mathrm{~d}$ & $2.80 \mathrm{~d}$ & $2.72 \mathrm{~b}$ \\
\hline $\mathrm{T}_{6}=\mathrm{NPK}$ same at $\mathrm{T}_{4}$ from urea, $\mathrm{TSP}$ and $\mathrm{MoP}$ & $3.18 \mathrm{c}$ & $3.04 \mathrm{c}$ & $2.87 \mathrm{~b}$ \\
\hline $\mathrm{T}_{7}=$ Absolute control & $2.13 \mathrm{e}$ & $2.02 \mathrm{e}$ & $2.38 \mathrm{~d}$ \\
\hline $\mathrm{T}_{8}=$ Only $\mathrm{N}$ & $2.51 \mathrm{~d}$ & $2.47 \mathrm{~d}$ & $2.51 \mathrm{~cd}$ \\
\hline
\end{tabular}

Figures in a column means followed by different letters differ significantly, whereas figure with common letter(s) are not significantly different at the $5 \%$ level by DMRT

Table 7. Effect of different treatments on the grain yield advantage over recommended fertilizer rate average of three locations during Boro, T Aman and T. Aus season

\begin{tabular}{|c|c|c|c|}
\hline Treatments & $\begin{array}{l}\text { Average yield } \\
\text { increase in Boro } \\
\text { over recommend } \\
\text { rate }(\%)\end{array}$ & $\begin{array}{c}\text { Average yield } \\
\text { increase } \\
\text { In T. Aman over } \\
\text { recommend rate } \\
(\%)\end{array}$ & $\begin{array}{l}\text { Average } \\
\text { yield } \\
\text { increase } \\
\text { over } \\
\text { recommend } \\
\text { rate }(\%)\end{array}$ \\
\hline $\begin{array}{l}T_{1}=\text { Rec. } \\
\text { fertilizer rate }\end{array}$ & 0.0 & 0.00 & 0.00 \\
\hline $\mathrm{T}_{2}=$ USG N$_{52} \mathrm{~kg} \mathrm{ha}^{-1}+$ Rec. PKSZn & 3.25 & 11.43 & 9.41 \\
\hline $\begin{array}{l}\mathrm{T}_{3}=\mathrm{NPK} \text { briquette } \mathrm{N}_{87} \text { (Boro) and } \mathrm{N}_{44} \text { (T. Aus and } \\
\text { T. Aman) }\end{array}$ & 10.06 & 5.47 & -1.85 \\
\hline $\mathrm{T}_{4}=3.40 \mathrm{~g}$ NPK briquette $\mathrm{N}_{57}$ & -10.51 & 27.99 & 17.69 \\
\hline $\mathrm{T}_{5}=\mathrm{NPK}$ same at $\mathrm{T}_{3}$ from urea, TSP and MoP & 1.82 & -4.45 & -10.97 \\
\hline $\mathrm{T}_{6}=\mathrm{NPK}$ same at $\mathrm{T}_{4}$ from urea, TSP and MoP & -9.89 & 3.49 & -2.02 \\
\hline $\mathrm{T}_{7}=$ Abs. control & -45.51 & -25.21 & -27.45 \\
\hline $\mathrm{T}_{8}=$ Only $\mathrm{N}$ & -8.04 & 14.50 & 16.83 \\
\hline
\end{tabular}

NPK briquette deep placement increased rice grain yield when compared with urea broadcast and incorporation during Boro season at three locations with equivalent $\mathrm{N}_{87}$ rate (Fig. 1) with same PK level. Similar yield increase was also observed in all locations in both T. Aus and T. Aman season (Fig. 2-5). In Figures 3 and 5-6, a single 3.4 g NPK briquette deep placement ( $\mathrm{N}_{57}$ rate) is compared with same rate of $\mathrm{N}, \mathrm{P}$, and $\mathrm{K}$ applied as urea, TSP and MoP for T. Aus, Aman and Boro season respectively. The NPK rates were low for the Boro rice; hence there was no difference between deep placement of NPK and the conventional application (Fig. 6). However, for both the T. Aus and T. Aman crop NPK briquette deep placement produced higher yield than conventional application of NPK (Fig. 3 and 5). The results indicate that the deep placement of NPK briquette has some yield advantage over urea, TSP, and MoP application at equivalent rate in wetland rice culture system. In Boro season deep placement of NPK briquette with $87 \mathrm{~kg}$ N/ha produced higher yield than the higher level of BRRI recommended $\mathrm{N}_{138}$ rate (Fig. 7). It means deep placement method saved on an overage 51-9-20 kg NPK/ha across location with increase of $10 \%$ rice yield in Boro season. Similarly, T. Aus and T. Aman season also showed 28 and 18\% yield increase with less fertilizer as NPK briquette deep placement as compared to higher level of BRRI recommended $\mathrm{N}$ rate (Figure 8-9). 


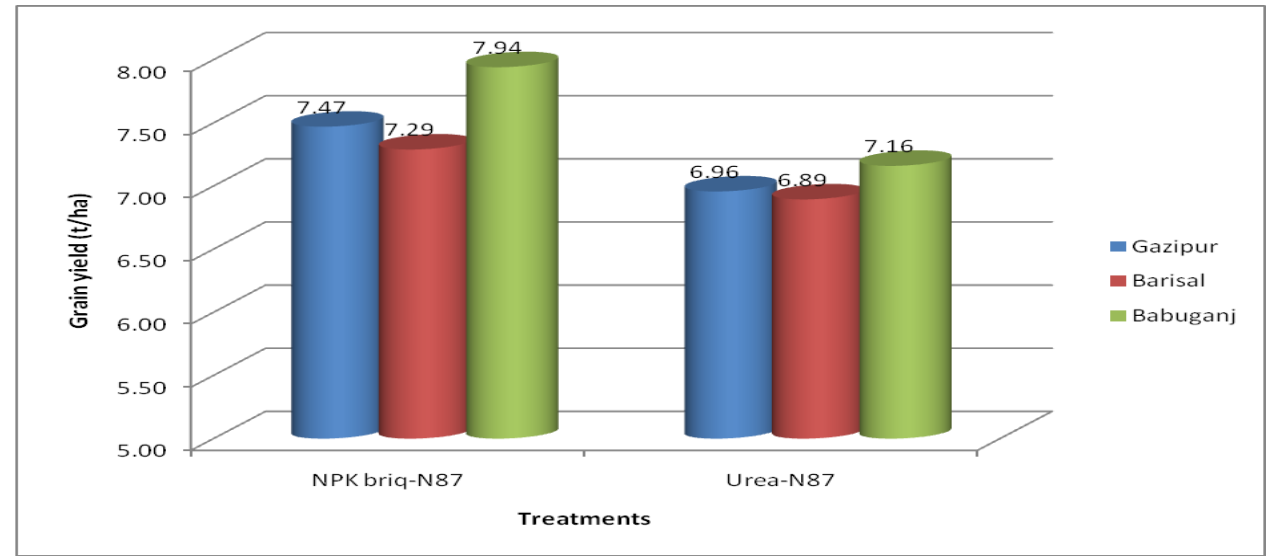

Fig. 1. Influence of NPK briquette and urea-N at equivalent rate $\left(\mathrm{N}_{87}\right)$ on the yield of BRRI dhan29 in Boro season at different locations.

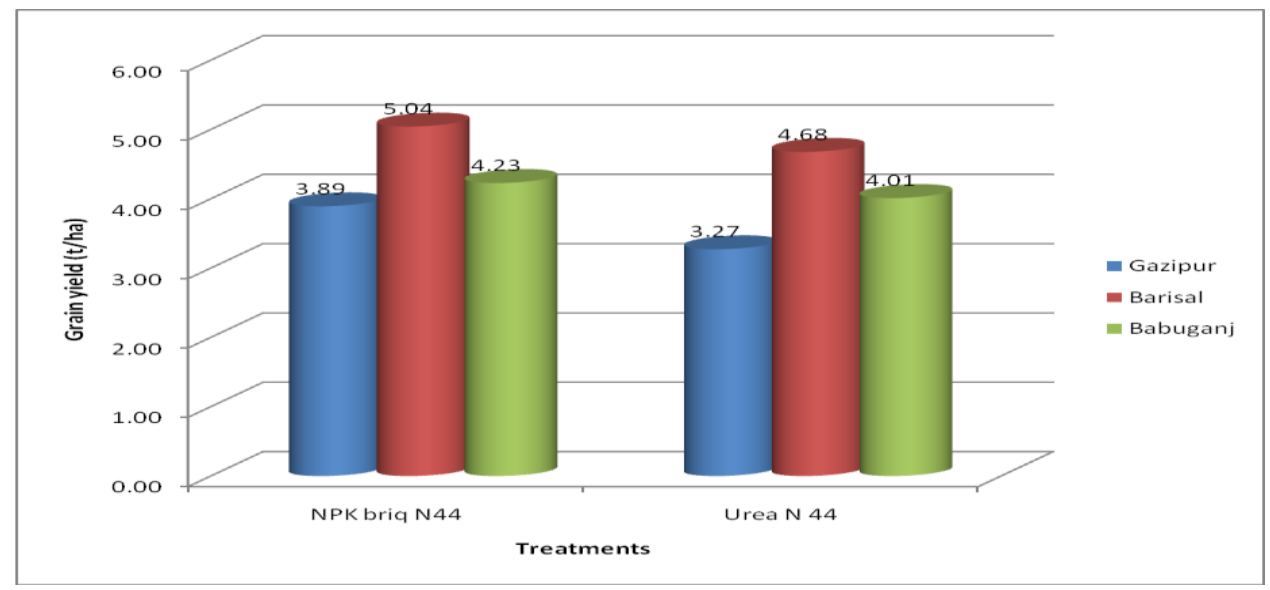

Fig. 2. Yield comparison between NPK briquette and urea-N at equivalent rate $\left(\mathrm{N}_{44}\right)$ at different locations in T. Aus season.

Again, deep placement method saved $\mathrm{N}$ fertilizer $20 \mathrm{~kg} / \mathrm{ha}$ in $\mathrm{T}$. Aus and $36 \mathrm{~kg} / \mathrm{ha}$ in $\mathrm{T}$. Aman season. Based on these results it may be concluded that NPK briquette deep placement in wet land rice culture enhanced rice yield with less amount of fertilizer than using the conventional method of urea, TSP and MoP application.

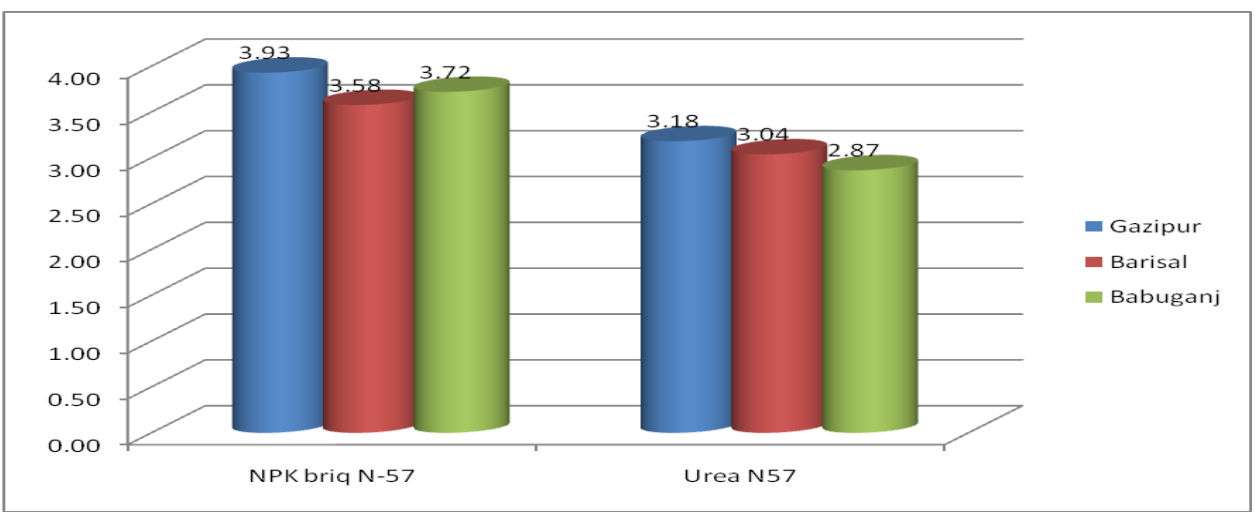


Fig. 3. Influence of NPK briquette and urea-N at equivalent rate $\left(\mathrm{N}_{57}\right)$ on the yield of BRRI dhan27 in T. Aus season at different locations.

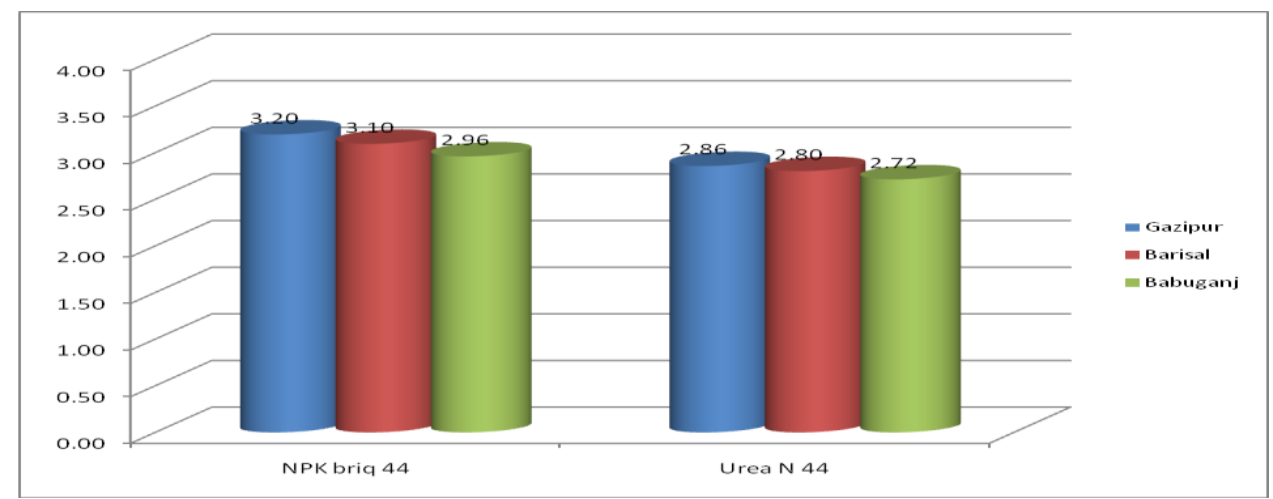

Fig. 4. Yield comparison between NPK briquette and urea-N at equivalent rate $\left(\mathrm{N}_{44}\right)$ at different locations in T Aman season.

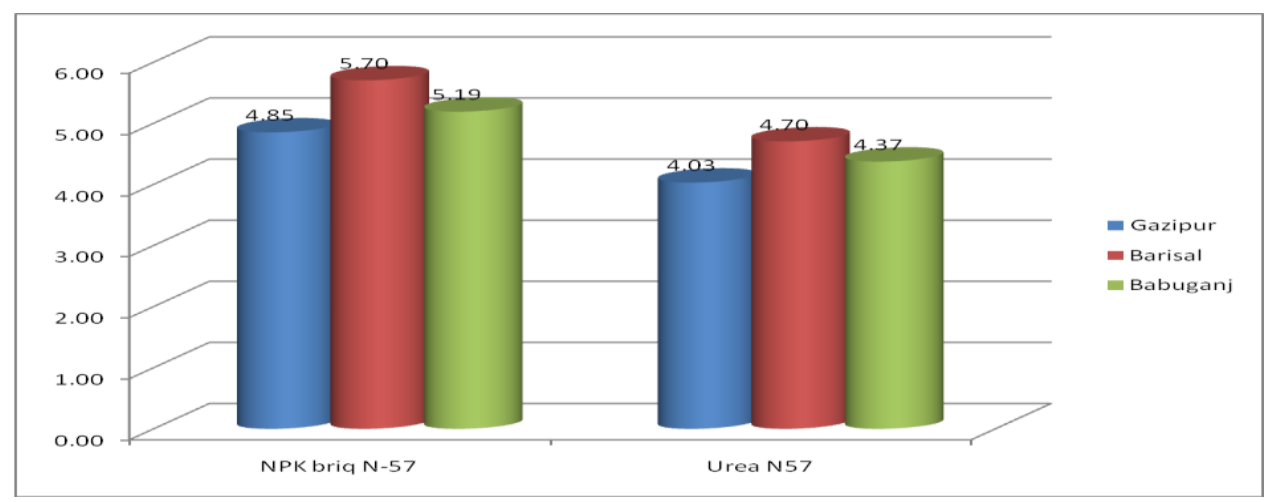

Fig. 5. Yield comparison between NPK briquette and urea-N at equivalent rate $\left(\mathrm{N}_{57}\right)$ on the yield of BRRI dhan49 at different locations in T Aman season.

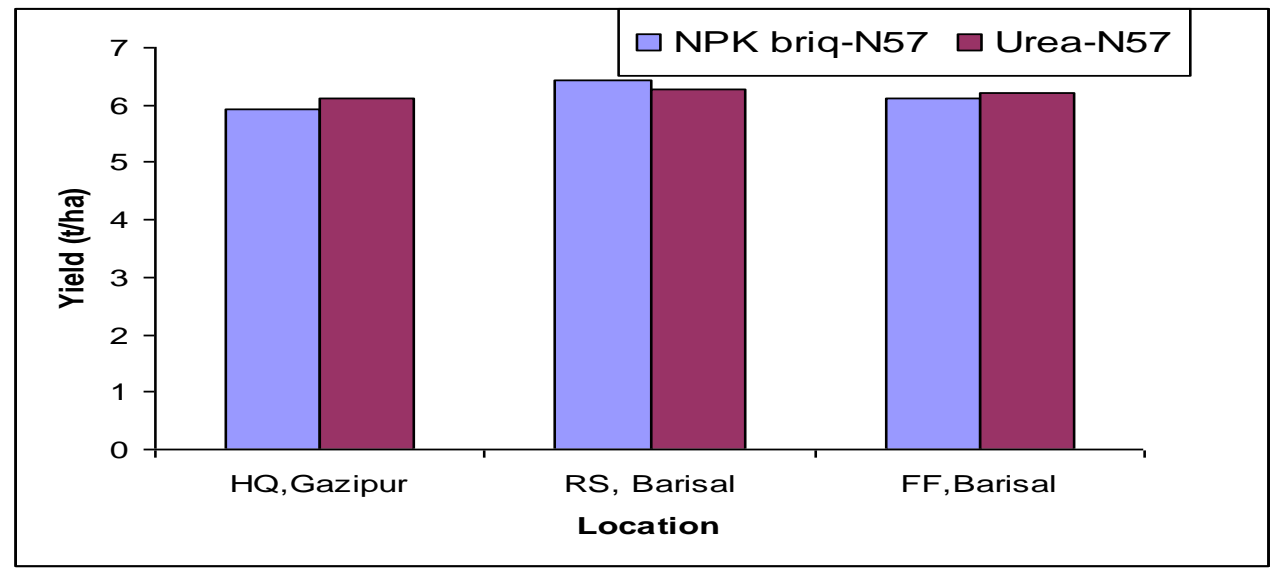

Fig. 6. Yield comparison between NPK briquette and urea-N at equivalent rate $\left(\mathrm{N}_{57}\right)$ with same level of PK on the yield of BRRI dhan29 in Boro season. 


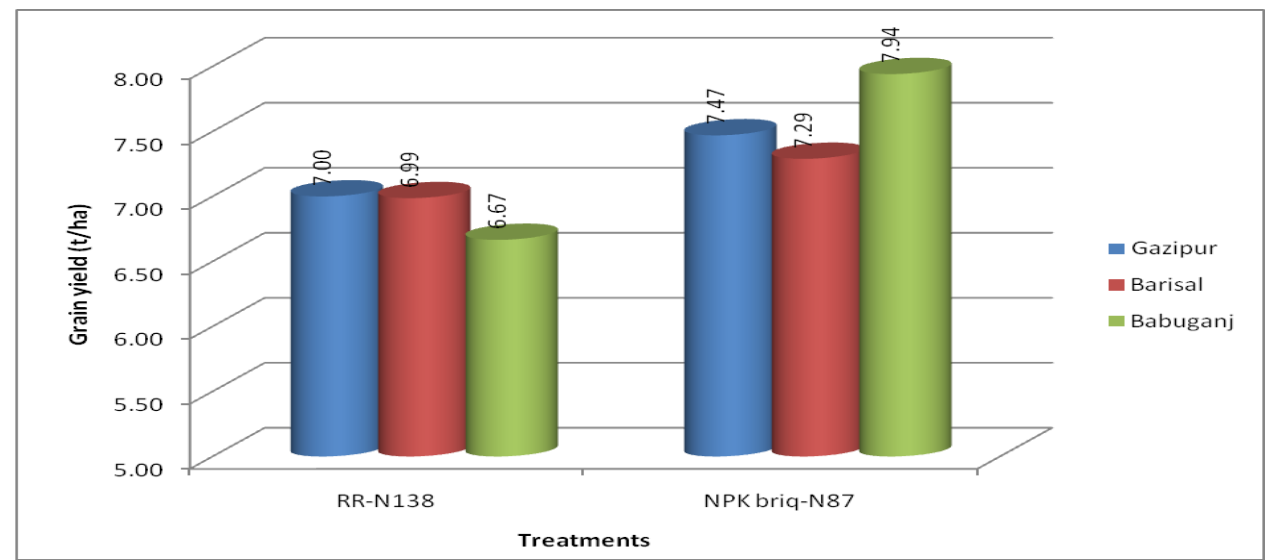

Fig. 7. Comparison of grain yield between recommended fertilizer rate $\left(\mathrm{N}_{138}\right)$ and NPK briquette $\left(\mathrm{N}_{87}\right)$ deep placement in Boro season at different locations.

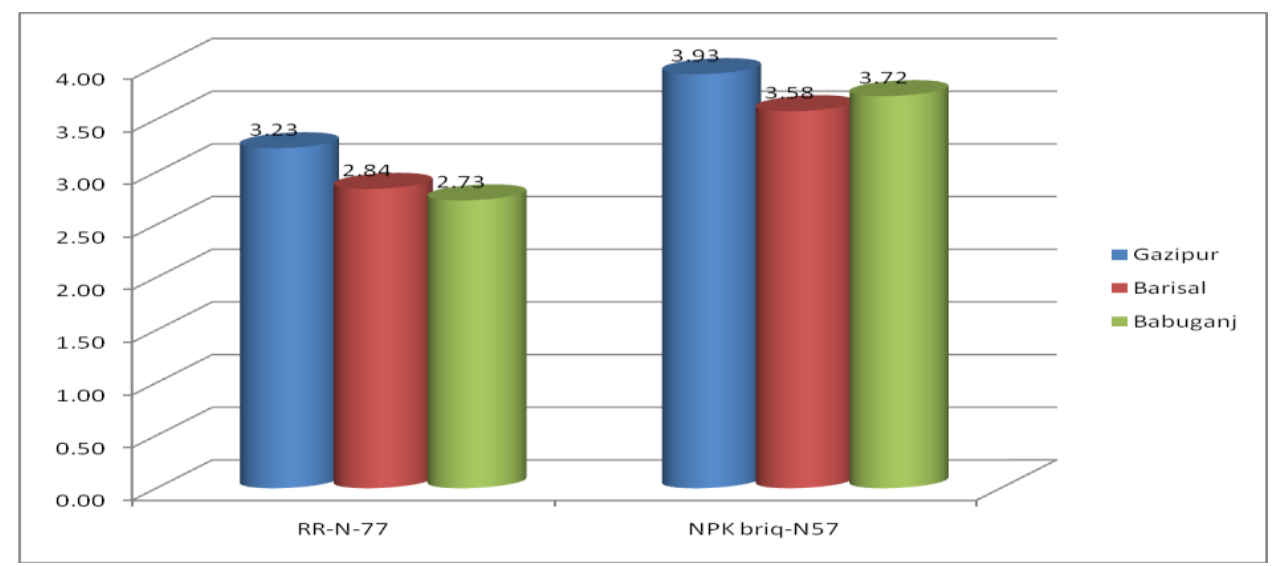

Fig. 8. Comparison of grain yield between recommended fertilizer rate $\left(\mathrm{N}_{77}\right)$ and NPK briquette $\left(\mathrm{N}_{57}\right)$ deep placement during $\mathrm{T}$ Aus season at different locations.

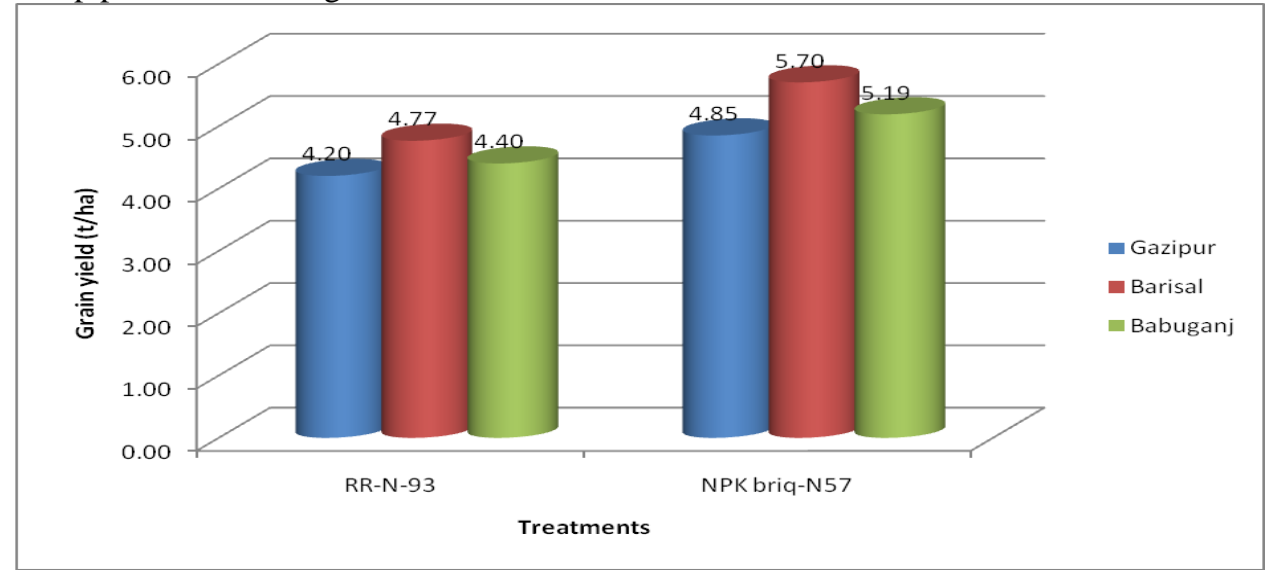

Fig. 9. Grain yield comparison between recommended fertilizer rate $\left(\mathrm{N}_{93}\right)$ and NPK briquette $\left(\mathrm{N}_{57}\right)$ deep placement during T Aman season at different locations. 
A partial budget analysis was done to determine the economic benefit of NPK briquette over urea broadcast and incorporation. Table 4 revealed that additional yields obtained from NPK briquette deep placement were 577,713 and $880 \mathrm{~kg} / \mathrm{ha}$ in Boro, T. Aus and T. Aman season, respectively and thus additional returns (Tk./ha) were Tk. 8655, Tk. 10695 and Tk. 13200, respectively. On the other hand, by using NPK briquette for rice production additional cost were incurred Tk. 3540, Tk. 3144 and Tk. 3144 in Boro, T. Aus and T. Aman production, respectively. Therefore, in all seasons, positive net returns were obtained from NPK briquette placement for rice cultivation. Table 7 also showed that undiscounted BCR (3.20) were highest in T. Aman production followed by T. Aus production (2.40). Finally, it may conclude that use of NPK briquette over urea broadcast and incorporation was economically viable and efficient for rice production.

Table 7. Partial Budget Analysis of NPK Briquette for rice production in different seasons over urea broadcast and incorporation

\begin{tabular}{lcccccccc}
\hline Season & $\begin{array}{c}\text { NPK } \\
\text { Briquette }\end{array}$ & $\begin{array}{c}\text { Additional } \\
\text { Yield (t/ha) }\end{array}$ & $\begin{array}{c}\text { Additional } \\
\text { Return } \\
\text { (Tk./ha) }\end{array}$ & $\begin{array}{c}\text { Additional Cost (Tk./ha) } \\
\text { Cost for }\end{array}$ & $\begin{array}{c}\text { Labor } \\
\text { Briquette } \\
\text { Manuf. }\end{array}$ & $\begin{array}{c}\text { Total } \\
\text { Rost } \\
\text { Requirement }\end{array}$ & $\begin{array}{c}\text { Net } \\
\text { Return } \\
\text { (Tk./ha) }\end{array}$ & $\begin{array}{c}\text { Undiscounted } \\
\text { BCR }\end{array}$ \\
\hline Boro & $\begin{array}{c}2.4 \mathrm{~g}: 87- \\
20-25\end{array}$ & 577 & 8655 & 1140 & 2400 & 3540 & 5115 & 1.44 \\
\hline T. Aus & $\begin{array}{c}3.4 \mathrm{~g}: 57- \\
15-22\end{array}$ & 713 & 10695 & 744 & 2400 & 3144 & 7551 & 2.40 \\
\hline T. Aman & $\begin{array}{c}3.4 \mathrm{~g}: 57- \\
15-22\end{array}$ & 880 & 13200 & 744 & 2400 & 3144 & 10056 & 3.20 \\
\hline
\end{tabular}

Note: Estimated price of paddy was Tk. 15/kg, Manufacturing cost of NPK briquette was Tk. 6/kg,

Labor required for NPK briquette placement was 8 man-day /ha and wage rate of labor was Tk. 300/day

Experimental result revealed that deep placement method of NPK briquette in rice production saved 51 $\mathrm{kg} \mathrm{N} / \mathrm{ha}$ in Boro and around $29 \mathrm{~kg} \mathrm{~N} / \mathrm{ha}$ in T. Aus and T. Aman season. Deep placement method favors to obtain higher yield compared to urea application to some extend. Undiscounted benefit cost ratio (BCR) was 1.44, 2.40 and 3.20 for Boro, T. Aus and T. Aman rice, respectively.

\section{Conclusion}

Deep placement of NPK briquettes $(2 \times 2.4 \mathrm{~g})$ increased about $10 \%$ higher rice grain yield and saved 51, 9 and $20 \mathrm{~kg} \mathrm{NPK} / \mathrm{ha}$ than BRRI fertilizer recommended rate in Boro season. Similarly, 23\% more rice yield obtained from NPK briquette $(1 \times 3.4 \mathrm{~g})$ use than BRRI recommended fertilizer rate with a savings of $28 \mathrm{~kg} \mathrm{~N}$ and $7 \mathrm{~kg} \mathrm{~K} / \mathrm{ha}$ irrespective of T. Aus and T. Aman season. NPK briquette use also promotes balance fertilization in rice production. Deep placement of NPK briquette leads to the accumulation of nutrients in the reduced zone of soil and subsequently these nutrients are utilized efficiently by rice plants for better growth and increased yield. This method leads to a significant decrease in flood water NH4-N content and the resultant effect decrease the load of volatilization and surface runoff and ultimately reduces environmental pollution and it may be considered as environment friendly technology.

\section{References}

AIS (Agriculture Information Service). 2008. Krishi Diary (In Bengali). Khamarbari, New Airport Road, Farmgate, Dhaka, Bangladesh. p.23.

Anonymous. 2008. Economic Advisory sub-division. Economic Division. Minist. Plan. Govt. People's Repub. Bangladesh. p.132.

Barber, S.A. 1976. Efficient fertilizer use. In: Patterson F. L ed. pp.13-29. Agronomic research for food. Madison. WI ASA special publication No. 26. Amer. Soc. Agron. 
BBS (Bangladesh Bureau of Statistics). 2007. Statistical Year Book of Bangladesh Bureau of Statistics, Stat. Div., Min. Plan., Govt. People's Repub., Bangladesh, Dhaka. pp.47-50.

Bhuiyan, N.I., A.L. Shah, M.A. Saleque and S.K. Zaman. 1988. Effect of N source and application method on dry season irrigated rice. IRRN 13(3): 28.

Bulbule, A.V., A.G. Durgude, V.S. Patil and Kulkarnil. 2008. Fertilization of low land transplanted rice through briquettes. Crop Res. 35(1\& 2): 1-5.

De Datta, S.K. and E.T. Craswell. 1982. Nitrogen fertilizer and fertilizer management in wet land rice soils. In rice research strategies for the future, International Rice Research Institute. Los Banos, Laguna, Philippines, pp.283-316.

Fan Xiao-hui, S.Yong-sheng, L. De-xi, Y. Lin-zhang and L. Jia-fa. 2006. Ammonia volatilization losses and 15N balance from urea applied to rice on a paddy field. J. Env. Sci. 18 (2): 299-303.

IRRI (International Rice Research Institute). 2006. Rice Yield ( $\left.\mathrm{t} \mathrm{ha}^{-1}\right)$, by country and geographical Region. World Rice Statistics. Intl., Rice Res. Inst. Los Banos, Laguna, Philippines. pp.1-8.

Kapoor, V., U. Singh, S.K. Patil, H. Magre, L.K. Shrivastava, V.N. Mishra, R.O. Das, Samadhiya, J. Sanabria and R. Diamond. 2008. Rice growth, Grain yield, and flood water nutrient dynamics as affected by nutrient placement method and rate. Agron. J. 100(3): 526-536.

Mitsui, S. 1955. Inorganic nutrition, Fertilization and soil amelioration for low land rice. $2^{\text {nd }}$ ed. Yokendo Press, Tokyo, Japan 105p.

Mohanty, S.K., U. Singh, V. Balasubramanian and K.P. Jha. 1999. Nitrogen deep-placement technologies for productivity, profitability, and environmental quality of rainfed lowland rice systems. Nutr. Cycl. Agro ecosys. 53: 43-57.

Prasad, M. and R. Prasad. 1983. Removal of nitrogen, phosphorus and potassium by rice-wheat double cropping system as affected by duration of rice variety, methods of planting rice and levels and sources of nitrogen. Plant Soil. 70: 287-295.

Radov, A.S., I.V. Pustovoi and A.V. Korolkov. 1985. Practical on Agrochemistry. Moscow: Agropromizgat. 312p. (Russian).

Sanchez, P.A. 1976. Properties and management of soils in the tropics. John Wiley and Sons Inc. 618p.

Shioiri, M. 1941. Denitrification in paddy soils. (In Japanese.) Kagaku (Science in Japan) 16: 104-116. 